\title{
A New Noise-Tolerant Dynamic Circuit Design with Enhanced PDP Performance under Low SNR Environment
}

\author{
You-Gang Chen, I-Chyn Wey, and An-Yeu (Andy) Wu \\ Graduate Institute of Electronics Engineering, National Taiwan University, Taiwan \\ Contact Email:Semic@access.ee.ntu.edu.tw
}

\begin{abstract}
As the supply voltage is scaling down, both SNR and the circuit noise immunity are reduced. In this paper, we develop a new isolated noise-tolerant technique to prevent the dynamic circuit from the noise interference. As compared with the state of the art design, the noise immunity can be enhanced by $1.5 \mathrm{X}$. For enhancing the noise-tolerance, we can save $81 \%$ power delay product (PDP) in severe low SNR environment. Moreover, the proposed circuit can achieve $81 \%$ and $39 \%$ energy saving as compared with the conventional domino circuit and twin-transistor design, respectively.
\end{abstract}

\section{INTRUDUCTION}

As process technology and supply voltage scale down, the device can be operated faster with good power efficiency. Besides, the threshold voltage is also reduced to maintain sufficient driving force but the noise margin will be reduced. Moreover, high transistor integration density, high operating frequency, and complex wire interconnection make the noise issues difficult to handle [1-3]. The noise sources in the VLSI circuit mainly include the power-supply noise, the crosstalk noise, and the fluctuations in device parameters due to process variations. These noise sources are more and more serious in very deep submicron process $[2,3]$. For those analyses in the literature, all the noise can be modeled as input noise to quantitatively analyze the noise influence in VLSI circuits [2, 4, $10]$.

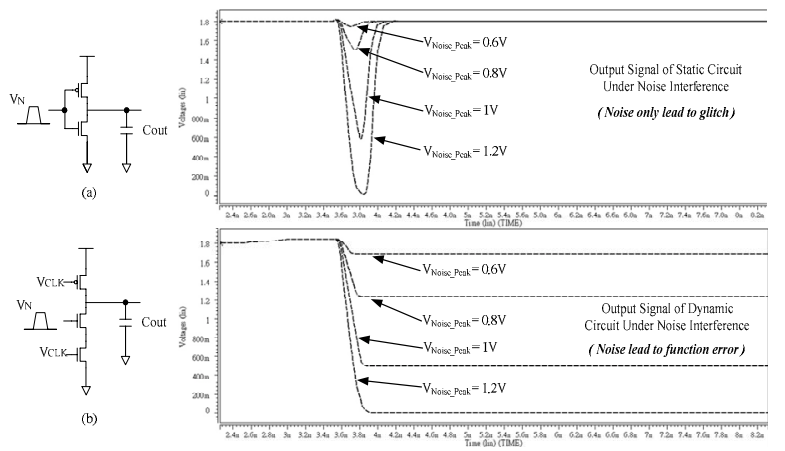

Fig. 1. Comparison of noise interference in dynamic circuits and static circuits

Dynamic circuit is one of popular logic families due to the high speed and performance characteristics. However, dynamic circuit is inherently susceptible to noise because of the floating situation occurs in the evaluation phase and the noise margin is just Vth (threshold voltage). Fig. 1 illustrates that dynamic circuits are more susceptible to noise as compared to static circuits. Under this condition, the dynamic circuit will have a logic failure but the static circuit only produces a glitch. Consequently, how to deal with the noise issue in dynamic circuits becomes the urgent design challenge. Therefore, in this paper, we develop a new noise-tolerant technique, called isolated noise-tolerant technique (INT) to prevent the noise interference in the dynamic circuit. With our proposed technique, the noise-tolerant mechanism will not be destroyed even if the operating environment is noise intensive. Therefore, the proposed design can have the noise immunity up to $1.5 \mathrm{X}$ enhancement.

\section{THE EXISTING NOISE TOLERANT DYNAMIC CIRCUIT DESIGNS}

Recently, the existing noise tolerant design techniques can be mainly divided into two parts. One is to prevent the dynamic node from floating $[5,6]$, and the other is to raise the source voltage to prevent the input gate from the noise injection [7-10] Preventing dynamic nodes from floating can be achieved by connecting the voltage source to the floating node or constructing the complementary p-network. However, this method is still limited the noise immunity to the upper bound as conventional static CMOS circuit.

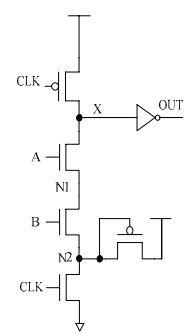

(a)

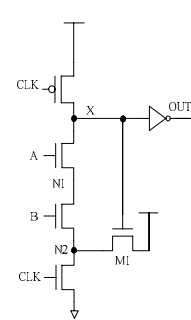

(b)

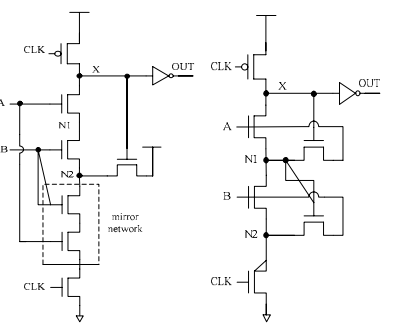

(c)

(d)
Fig. 2. Comparison of different noise tolerant techniques: (a)pMOS pull-up(b)nMOS pull-up (c)mirror (d)twin

Raising the source voltage of pull-down network can increase the transistor threshold voltage. In Fig. 4(a) [7], it uses a pull-up device to increase the source voltage of NMOS to increase the noise threshold. However, such connection will lead to large dc power consumption and the output signal may be non-full-swing. Fig. 4(b) [8] shows another approach by employing an nMOS pull-up transistor with a feedback-control. Moreover, this technique has the same problem as the Fig. 4(a). Fig. 4(c) [9] shows the mirror technique, which can solve both dc power consumption and non-full-swing of output signal problems. The mirror technique uses a mirror nMOS pull-down network to increase the dynamic switching threshold with the principle of Schmitt trigger. However, the stacked nMOS transistor will seriously cause the critical path. Recently, the twin-transistor technique employs an extra transistor for every transistor in the pull-down network to pull up the source potential and enhances the noise-tolerant ability in an active manner. As shown in Fig. 4(d) [10], the drain nodes of the pull-up nMOS transistors are connected to the inputs. By this way, the charge injected by noise can be effectively discharged through this additional pull-up nMOS. Moreover, the source potential of pull-down network can also be raised along with the increasing of noise amplitude. Consequently, the noise tolerant ability of dynamic circuits can be greatly improved. However, the noise-tolerant mechanism may still possibly be destroyed by the input noise. Moreover, this technique is not suitable for the $(\mathrm{A}+\mathrm{B}) \cdot \mathrm{C}$ type logic 
because of the existence of direct conducting path.

No matter how to enhance the noise immunity by preventing the dynamic nodes from floating or by raising the source voltage of pull-down nMOS transistors, the noise-tolerant mechanisms all are not under protection; therefore, the noise-tolerant mechanism may possibly be destroyed by the noise interference. Consequently, all these existing techniques must still pay expensive design penalty in terms of speed, power, and area to achieve the noise tolerant goal. Especially as the requirement of noise tolerance is increased along with the progress of process technology, the design overhead will further increase dramatically.

\section{THE PROPOSED ISOLATED NOISE-TOLERANT DYNAMIC CIRCUIT TECHNIQUE}

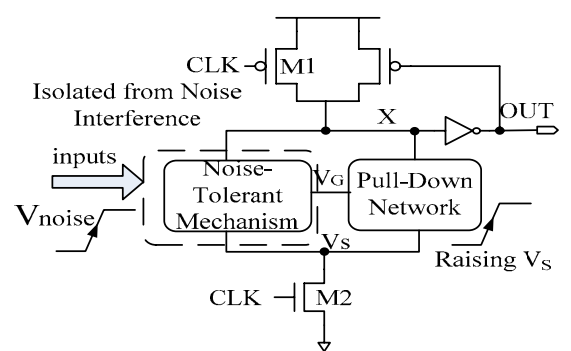

(a)

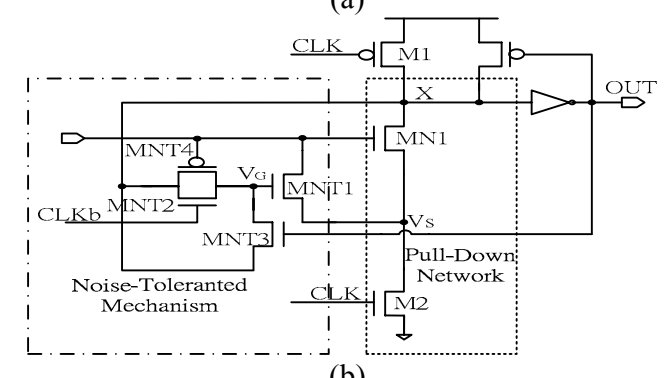

(b)

Fig. 3. Proposed isolated noise-tolerant technique: (a) general circuit schematic, (b) buffer schematic

\section{A. Characteristics of the Proposed INT Technique}

Fig. 3(a) is the proposed isolated noise-tolerant (INT) technique and it concludes a noise isolated mechanism and an original pull-down network. Fig. 3 (b) is proposed buffer circuit, MNT1 (NT-transistor) provides a path to transmit noise to raise the source voltage and enhances the noise immunity. MNT2 is utilized to precharge node $V_{G}$ to make MNT1 conduct and turned off to protect the noise-tolerant mechanism from destroyed in the evaluation phase; MNT3 can provide a discharge path for maintaining function correction; MNT4 is utilized to prevent the occurrence of voltage overshooting. The weak feedback keeper can assist in preventing the dynamic node from floating. The main difference between the proposed INT technique and conventional noise-tolerant techniques is that the noise-tolerant mechanism in the INT circuits can be protected from being destroyed even if the noise input is enormous.

\section{B. Operation of the Proposed Noise-Tolerant Technique}

The operation of the proposed circuits can be separated into pre-charge phase and evaluation phase. To simplify the explanation of operation in the proposed noise-tolerant circuit, we take the dynamic buffer circuit as example. The same operation principle can be popularized to all other dynamic circuits.

\section{1) Pre-charge Phase:}

In the pre-charge phase, the clock signal CLK is low and the inverse clock signal $\mathrm{CLKb}$ is high. The dynamic node $\mathrm{X}$ is charged to logic high and the output node OUT is logic low. In this phase, Fig.4(a) is a simple illustration of the buffer circuit, node $\mathrm{V}_{\mathrm{G}}$ in the buffer circuit is precharged to high to make MNT1 conduct because MNT2 is turned on by CLKb and MNT3 is turned off by OUT. The conduction of MNT1 is essential for raising the source voltage of MN1 to enhance the noise-tolerance in evaluation phase.

2) Evaluation Phase:

In the evaluation phase, CLK is switched to high and CLKb is switched to low. The dynamic node X is floating because MP1 is turned off by CLK; therefore, the noise-isolated noise-tolerant mechanism must be activated in this phase to prevent the floating node $\mathrm{X}$ from being disturbed by the noise. As the input A is logic " 0 ", the noise-tolerant mechanism is activated by MNT2 and MNT3. Fig. 4(c) is a simple illustration, MNT2 will be turned off as CLKb switches to low and MNT3 will also be turned off since the input A is logic " 0 "; therefore, node $\mathrm{V}_{\mathrm{G}}$ is isolated and latched in logic high to maintain the conduction of MNT1. As noise interrupts the circuit as illustrated in Fig.4 (b), the noise charge can be drained away immediately through MNT1 to node $\mathrm{V}_{\mathrm{S}}$ and the source voltage of MN1 will be raised by the disturbing signal. Hence, the noise-tolerant ability can be enhanced. Because the noise-tolerant ability is enhanced by raising the source voltage of MN1; therefore, it is important to maintain the conducting situation of MNT1 to achieve good noise immunity. In the evaluation phase of proposed circuit, the conduction situation of MNT1 can be hold because the node $V_{G}$ is isolated. Therefore, the noise-tolerant mechanism can be protected and the noise-tolerant ability will not degrade even under enormous noise interference. As the input A is logic " 1 ", the node $\mathrm{X}$ will be pull-down to logic low and OUT is logic high. As a result, the charge in the node $\mathrm{V}_{\mathrm{G}}$ can be discharged to turn off MNT1 to ensure the correctness of function in normal mode, as illustrated in Fig. 4(d).
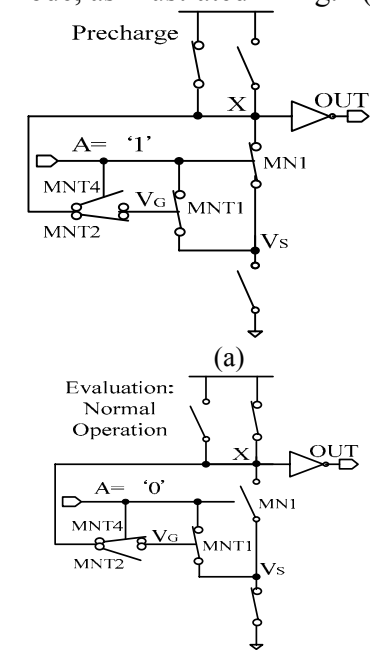

(c)

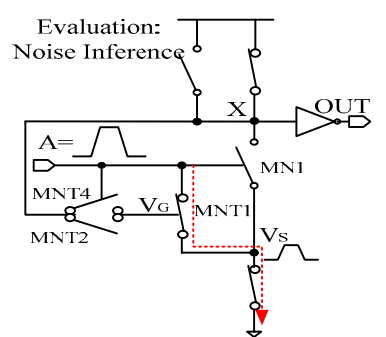

(b)

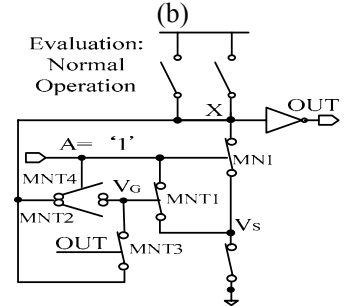

(d)
Fig. 4. The operation of the proposed noise-tolerant circuit: (a) operate in pre-charge phase (b) in evaluation phase with noise interference operates in evaluation phase. (c) normal operation in evaluation phase as input signal is " 0 " (d) normal operation in evaluation phase as input signal is " 1 "

\section{Noise-Isolated Noise-Tolerant Property}

The gate voltage of noise-tolerant transistor (NT-transistor) dominates the capability of noise-tolerance. The degradation of gate voltage results in the descent of noise-tolerant ability. In the conventional noise-tolerant techniques, the gate voltage of noise-tolerant transistor usually 
degrades as the interference of noise goes serious. As illustrated in Fig. 5(a), the gate voltage of noise-tolerant transistor degrades quickly as the injecting noise is high enough to turn the pull-down nMOS transistor on. The degradation of gate voltage will further aggravate the interference of noise because the noise tolerant mechanism is destroyed. Preventing the degradation of gate voltage can preserve the ability of draining the noise charge away. In the proposed design, the gate voltage of noise-tolerant transistor MNT1 can be isolated from noise interference; therefore, the noise-tolerant mechanism can be prevented from been destroyed. As illustrated in Fig. 5(a), the gate voltage of MNT in the proposed circuit will not degrade even if the injecting noise is enormous. The corresponding source voltage $(\mathrm{Vs})$ is illustrated in Fig. 5(b). From Fig. 5(b), we can see that the source voltage is raised in a noise-dependent manner in active style noise-tolerant circuits. Moreover, the source voltage can still be raised even under serious noise interference. Therefore, in the proposed design, the noise-tolerant ability can be greatly improved

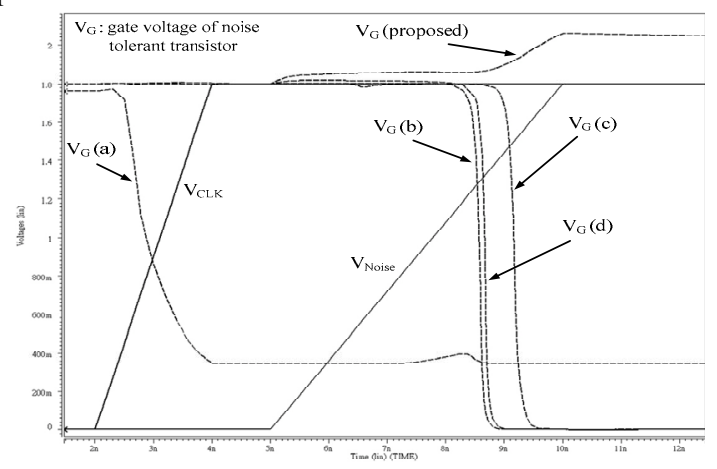

(a)

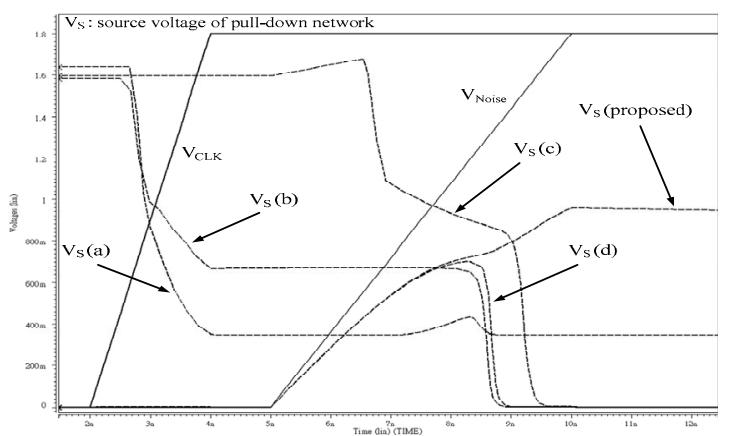

(b)

Fig. 5. The analysis of (a) gate voltage and (b) source voltage of the noise tolerant transistor with the injecting noise.

\section{PERFORMANCE ANALYSIS OF NOISE-TOLERANT DYNAMIC CIRCUIT TECHNIQUE}

In this session, we will discuss the trade-off between the noise-tolerant enhancement and performance overhead. The performance comparison results of various noise-tolerant techniques in dynamic circuits are based on TSMC $0.18 \mathrm{um}$ process technology. In the 2-input AND gate, the input pattern is applied based on the consideration of the worst case of noise interference, while input A is logic " 1 " and input B initially is logic " 0 ".

In the following, we will further take the noise duration into consideration, we can use the noise immunity curves (NICs) $[1,5,9,10]$ to compare the performance of various noise-tolerant techniques. This curve is the locus of amplitude $\left(\mathrm{V}_{\text {noise }}\right)$ and noise duration $\left(\mathrm{T}_{\text {noise }}\right)$ combinations. By using the
NIC, we can distinguish the noise-tolerant ability of different design styles. The upper curves in Fig. 6 represent the circuit can operate in noisier environment. As compared with the state of the art design, the noise immunity can be enhanced by $1.5 \mathrm{X}$. To quantify the information in the NIC, average noise threshold energy (ANTE) $[1,5,9,10]$ is a convenient metric that can be directly derived from the NIC by averaging the energy of noise pulses that cause the function error and it can be normalized to NANTE. Table 1 shows the comparison results of ANTE and NANTE, the proposed circuit is more energy efficient and expends $39-81 \%$ less energy.

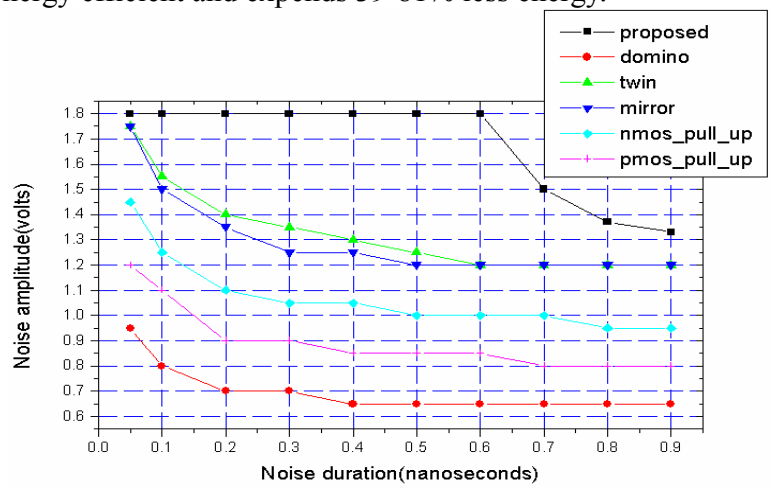

Fig.6. Noise immunity curves (NICs)

Table 1 ANTE comparison

\begin{tabular}{|l|c|c|c|}
\hline & ANTE & NANTE & Energy Saving \\
\hline proposed( $\mathrm{V}^{2}$-ns $)$ & 1.170 & 1 & \\
\hline domino( $\mathrm{V}^{2}$-ns $)$ & 0.200 & 5.84 & $81 \%$ \\
\hline twin( $\left(\mathrm{V}^{2}\right.$-ns $)$ & 0.704 & 1.66 & $39 \%$ \\
\hline mirror( $\left(\mathrm{V}^{2}\right.$-ns $)$ & 0.687 & 1.70 & $41 \%$ \\
\hline nmos_pull_up & 0.460 & 2.53 & $60 \%$ \\
\hline pmos_pull_up & 0.321 & 3.63 & $72 \%$ \\
\hline
\end{tabular}

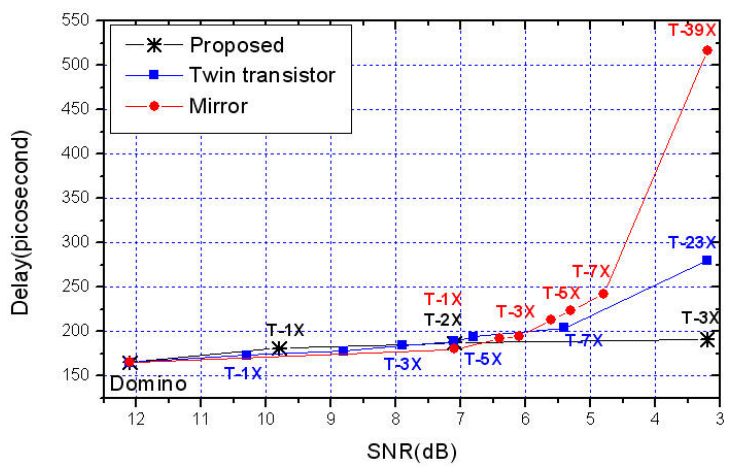

Fig. 7. The trade-off of the noise threshold against delay

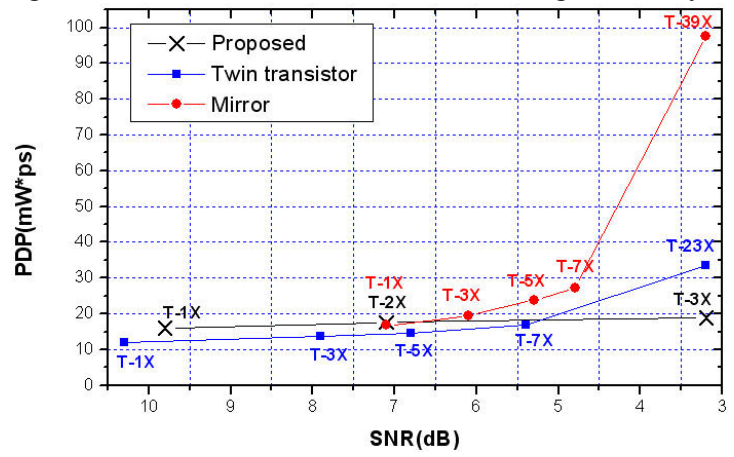

Fig. 8. The trade-off of the noise threshold against PDP

As the size of NT-transistor using to raise the source voltage increases, the NICs can be raised up to perform better noise immunity. However, the design overhead will also 
increase; especially the delay time of dynamic circuits will be postponed. We compare the delay time while applying different noise-tolerant techniques in Fig. 7. As shown in Fig. 7, the mirror technique which is a kind of the passive mode noise-tolerant technique must spend expensive design overhead up to 39X NT-transistor size to operate accurately under severely noisy signal. However, as shown in Fig. 7, the proposed design can easily provide low design overhead against noise, while the twin-transistor technique must pay $23 \mathrm{X}$ extra size effort and $0.7 \mathrm{X}$ speed penalty. In other words, under the same performance sacrifice of speed, the proposed design has better noise tolerant ability. In Fig. 8, we compare the performance penalty in terms of power-delay-product (PDP) for enhancing the noise tolerance. The proposed design provides evident superiority in saving the performance penalty as compared with the twin and mirror techniques. In the very noisy environment which the SNR is low to $3.2 \mathrm{~dB}$, the proposed design can save $46 \%$ and $81 \%$ performance penalty in PDP.

\section{CHIP IMPLEMENTATION}

We implement the proposed noise-tolerant technique in the Manchester adder to demonstrate the performance of noise-tolerance and verify the function in the presence of noise. Fig.9 is the carry generation circuit with the proposed INT mechanism which shows in the dash line block and the dash-line transistors are used to solve the direct conducting path problem. Fig. 10 is the chip layout of the total area without pad is $0.68 * 0.15 \mathrm{~mm}^{\wedge} 2$. Chip performance is summarized in Table 2. Comparing with different noise-tolerant technique, the proposed design has a lower delay time and PDP. Moreover, in the seriously noisy environment, the proposed design uses only $3 X$ NT-size to operate accurately. The carry-out waveform of the Manchester adder using the proposed and the conventional design is shown in Fig. 11. We input the AWGN noise interference to the adder and the waveform reveals that the noise tolerant technique can effectively immunize the adder from the noise interference.

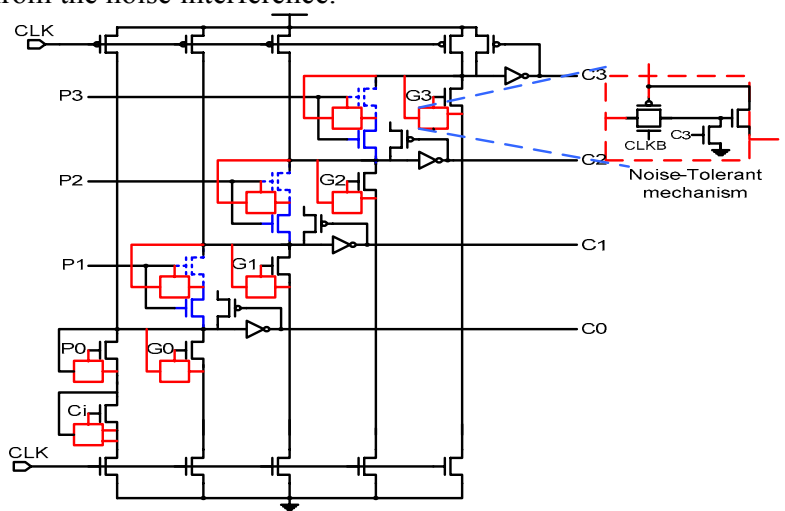

Fig. 9. The proposed noise-tolerant technique implemented in Manchester adder

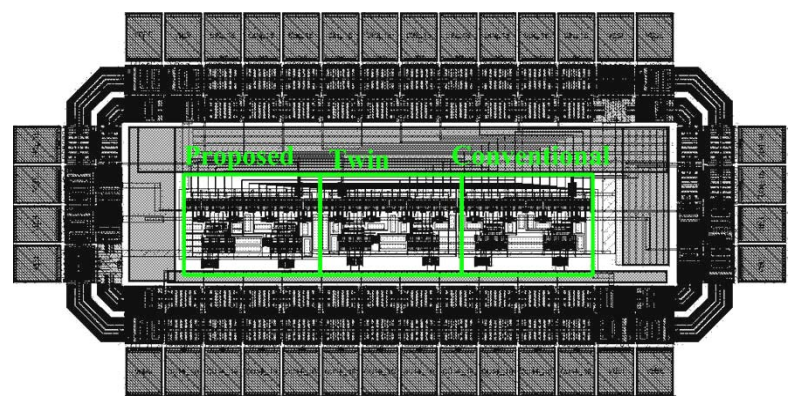

Fig. 10. Chip layout
Table 2 Performance Summary

\begin{tabular}{|c|c|c|c|c|}
\hline Implementation Process & \multicolumn{4}{|c|}{ TSMC 0.18um } \\
\hline Supply Voltage & \multicolumn{4}{|l|}{$1.8 \mathrm{~V}$} \\
\hline Clock Frequency & \multicolumn{4}{|l|}{$100 \mathrm{MHz}$} \\
\hline Design Type & Domino & Mirror[8 & Twin[9] & Propose \\
\hline Transistor Count & 1128 & 7 & 1170 & 1206 \\
\hline Circuit Area $\left(\mathrm{mm}^{\wedge} 2\right)$ & 0.102 & & 0.157 & 0.183 \\
\hline ANTE $\left(V^{2}\right.$-ns $)$ & 0.269 & & 0.565 & 1.077 \\
\hline Delay Time @ $\mathrm{SNR}=3.2 \mathrm{~dB}$ & 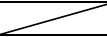 & $517 \mathrm{ps}$ & $280 \mathrm{ps}$ & $191 \mathrm{ps}$ \\
\hline Delay Time @ $\mathrm{SNR}=5 \mathrm{~dB}$ & - & 230 & 220 & 180 \\
\hline PDP @SNR=3.2dB(mw*ps) & & 97.6 & 33.5 & 18.9 \\
\hline PDP @ SNR=5dB(mw*ps) & & 25 & 27.3 & 18 \\
\hline NT-Size @SNR=3.2dB & & $39 x$ & $23 x$ & $3 x$ \\
\hline NT-Size @ SNR=5dB & & $6 \mathrm{x}$ & $8 \mathrm{x}$ & $2.5 \mathrm{x}$ \\
\hline
\end{tabular}

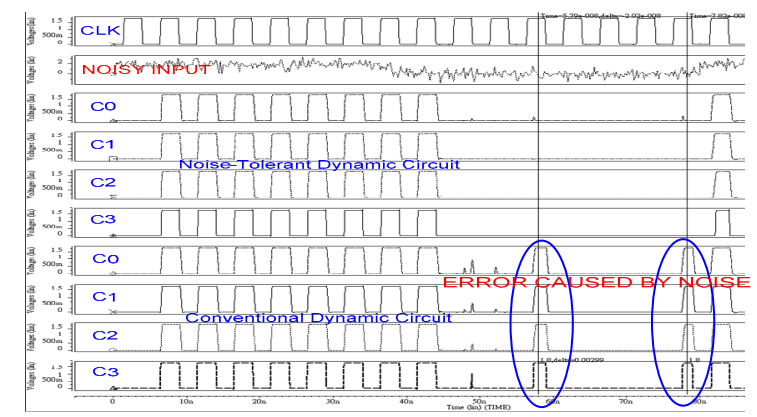

Fig. 11. Simulation Waveform in Manchester adder (a) employs the proposed noise-tolerant technique. (b) uses the conventional dynamic adder

\section{CONCLUSIONS}

In this paper, a high noise-immunity and energy efficiency design technique for dynamic circuits is presented. Comparing with the state of the art design, the noise immunity can be enhanced up to $1.5 \mathrm{X}$. In the low SNR (3.2dB) environment, the proposed design can save $81 \%$ performance penalty in PDP.

\section{REFERENCES}

[1] N. R. Shanbhag, "Reliable and efficient system-on-chip design," IEEE Computer Magazine, vol 37, pp. 42 -50, Mar. 2004.

[2] P. Larsson and C. Svensson, "Noise in Digital Dynamic CMOS Circuits", IEEE Journal of Solid-State Circuits, vol.29, pp.655-662, Jun.1994.

[3] K. L. Shepard and V. Narayanan, "Noise in deep submicron digital design," in Proc. Int. Conf. Computer Aided Design, 1996, pp.524-531.

[4] M. H. Chowdhury and Y. I. Ismail, "Analysis of Coupling Noise and Its Scalability in Dynamic Circuits," in Proceeding of IEEE Custom Integrated Circuits Conference, pp. 505- 508, Oct. 2004.

[5] L. Ding and P. Mazumder, "On circuit techniques to improve noise immunity of CMOS dynamic logic," IEEE Transactions on Very Large Scale Integration (VLSI) Systems, vol 12, pp. 910 - 925, Sept. 2004.

[6] A. Alvandpour, R. K. Krishnamurthy, K. Soumyanath, and S.Y. Borkar, "A conditional keeper technique for sub-0.13u wide dynamic gates," in Proceeding of International Symposium on VLSI Circuits, 2001, pp. 29-30.

[7] G. P. D'Souza, "Dynamic logic circuit with reduced charge leakage," U.S. Patent 5483 181, Jan. 1996.

[8] E. B. Schorn, "NMOS charge-sharing prevention device for dynamic logic circuits," U.S. Patent 5838 169, Nov. 1998.

[9] L. Wang and N. R. hanbhag, "Noise-tolerant dynamic circuit design," in Proceeding of International Symposium on Circuits and Systems, 1999, pp. I 549-552.

[10] G. Balamurugan and N.R. Shanbhag, "The twin-transistor noise-tolerant dynamic circuit technique," IEEE J. Solid-State Circuits, vol. 36, no. 2, pp. 273-280, Feb. 2001. 